\title{
Estimation of reproduction number and probable vector density of the first autochthonous dengue outbreak in Japan in the last 70 years
}

\author{
Hiroyuki Furuya ${ }^{1}$
}

Received: 12 May 2015/Accepted: 5 August 2015/Published online: 23 August 2015

(C) The Japanese Society for Hygiene 2015

\begin{abstract}
Objectives The first autochthonous case of dengue fever in Japan since 1945 was reported on August 27, 2014. Infection was transmitted by Aedes albopictus mosquitoes in Tokyo's Yoyogi Park. A total of 65 cases with no history of overseas travel and who may have been infected around the park were reported as of September 5, 2014. To quantify infection risk of the local epidemic, the reproduction number and vector density per person at the onset of the epidemic were estimated.

Methods The estimated probability distribution and the number of female mosquitoes per person (MPP) were determined from the data of the initial epidemic.

Results The estimated distribution $R_{0 \mathrm{i}}$ for the initial epidemic was fitted to a Gamma distribution using location parameter 4.25 , scale parameter 0.19 , and shape parameter 7.76 with median 7.78 and IQR (7.21-8.40). The MPP was fitted to a normal distribution with mean 5.71 and standard deviation 0.53 .

Conclusions Both estimated reproduction number and vector density per person at the onset of the epidemic were higher than previously reported values. These results indicate the potential for dengue outbreaks in places with elevated vector density per person, even in dengue nonendemic countries. To investigate the cause of this outbreak, further studies will be needed, including assessments of social, behavioral, and environmental factors that may
\end{abstract}

Hiroyuki Furuya

furuya@is.icc.u-tokai.ac.jp

1 Basic Clinical Science and Public Health, Tokai University School of Medicine, 143 Shimokasuya, Isehara-shi, Kanagawa 259-1193, Japan have contributed to this epidemic by altering host and vector conditions in the park.

Keywords Dengue - Epidemic curve Mathematical modeling · Urban · Behavioral and environmental factors

\section{Introduction}

A dengue epidemic was observed in western Japan from 1942 to 1945, and imported dengue cases subsequently have been reported in Japan [1]. Recently, there has been an increase in the number of imported dengue cases, with 249 such cases reported in 2013. A German tourist was suspected to have been infected with dengue while in Japan in August 2013, and the risk of autochthonous dengue in Japan was suggested [2].

The first autochthonous case of dengue fever in Japan since 1945 was reported on August 27, 2014, reflecting transmission by Aedes albopictus mosquitoes in Tokyo's Yoyogi Park $\left(540,529 \mathrm{~m}^{2}\right)$ [3]. A total of 159 cases with no history of overseas travel were reported through October 15,2014 , and 127 cases of these infections may have occurred at Yoyogi Park [4]. On August 28, the Tokyo metropolitan government sprayed pesticide in part of the park to kill the vector; traps were placed in 10 locations throughout the park to identify dengue virus-infected mosquitoes. Infected mosquitoes were detected from 4 of the 10 sites, and the metropolitan government closed the northern part of the park on September 4, 2014 [3].

In this study, the reported number of symptomatic cases was used to estimate the reproduction number and vector density per person at the onset of the epidemic, in an attempt to clarify potential mechanisms of dengue control in non-epidemic urban environments. 


\section{Materials and methods}

This study received ethical approval from the institutional review board (IRB) of Tokai University School of Medicine (No.14-331, September 16, 2014).

\section{Case reports and turning points}

Dengue fever cases were confirmed by the Ministry of Health, Labor, and Welfare and the governments of the prefectures where the cases lived. Case data included the reported date of onset, possible infection date and place, history of mosquito bites, residential prefecture, and diagnosing hospital prefecture [4].

To detect the turning point that was defined as the points in time at which the rate of accumulation cases changed from increasing to decreasing or vice versa, the cumulative number of cases on a logarithmic scale against the days from August 12 to September 3, 2014 was investigated.

\section{Estimation of the reproduction number $R_{0 \mathrm{i}}$ at the start of the epidemic}

The initial growth in the number of cases is considered to be exponential with parameter $\lambda$. The estimated reproduction number $R_{0 \mathrm{i}}$ at the start of the epidemic is calculated by the following equation, modified from the method of Massad et al. [5, 6].

$R_{0 i}=(1+\lambda / \gamma)(1+\lambda / \mu) \exp \left(\lambda\left(\tau_{\mathrm{e}}+\tau_{\mathrm{i}}\right)\right)$.

In this equation, $\tau_{\mathrm{i}}$ is the intrinsic incubation period; $\gamma$ is the inverse of the duration of the infectious period of the host; $\tau_{\mathrm{e}}$ is the extrinsic incubation period; and $\mu$ is the daily mortality rate of the vector. The parameter $\lambda$ is obtained as the slope of the linear phase of the curve of the daily number of new cases plotted against the cumulative number of cases. The extrinsic incubation period $\tau_{\mathrm{e}}$ is calculated as the temperature-dependent development rate of dengue virus in the vector; the value of $\tau_{\mathrm{e}}$ is determined using the following expression [1].

$\tau_{\mathrm{e}}=\frac{\mathrm{RHO} 25(T+273) / 298 \exp [(\mathrm{HA} / R)(1 / 298)-1 /(T+273)]}{1+\exp [(\mathrm{HH} / R) 1 /(\mathrm{TH}+273)-1 /(T+273)]}$.

The parameter RHO25 is the development rate at $25{ }^{\circ} \mathrm{C}$ in the absence of temperature inactivation of the enzyme; $\mathrm{HA}(\mathrm{cal} / \mathrm{mol})$ is the enthalpy of activation of the reaction that is catalyzed by the critical enzyme; $\mathrm{HH}$ (cal/mol) is the enthalpy change with high-temperature inactivation of the enzyme; TH $\left({ }^{\circ} \mathrm{C}\right)$ is the temperature at which $50 \%$ of the enzyme is inactivated at high temperature; $R$ is the universal gas constant $(1.987 \mathrm{cal} / \mathrm{mol}$ per degree $)$; and $T\left({ }^{\circ} \mathrm{C}\right)$ is the temperature. The values for $\mathrm{RHO} 25, \mathrm{HA}, \mathrm{HH}$, and
TH in A. albopictus were 0.080616, 15000, $6.203 \times 10^{21}$, and $-2.176 \times 10^{30}$, as provided in a previous study [1].

The daily mortality rate $\mu$ of the vector is calculated via the following equation:

$\mu=-\ln \left(0.91 \lambda_{1}(T) \lambda_{2}\left(P_{\mathrm{vd}}\right)\right)$,

where $T\left({ }^{\circ} \mathrm{C}\right)$ is the temperature and $P_{\mathrm{vd}}$ the vapor pressure deficit in mbar, approximated in terms of the temperature and the relative humidity $\mathrm{RH}$ [7]:

$P_{\mathrm{vd}}=6.11 \exp \left(17.3 \frac{T}{T+237.3}\right)(1-\mathrm{RH} / 100)$.

The function $\lambda_{1}$ and $\lambda_{2}$ are as follows:

$\lambda_{1}(T)= \begin{cases}(T-5) / 5 & \text { if } 5<T<10 \\ 1 & \text { if } 10<T<41 \\ 1-(T-41) / 2 & \text { if } 41<T<43 \\ 0 & \text { for other temperatures }\end{cases}$

$\lambda_{2}\left(P_{\mathrm{vd}}\right)= \begin{cases}1 & \text { if } P_{\mathrm{vd}}<10 \\ 1-0.4\left(P_{\mathrm{vd}}-10\right) / 20 & \text { if } 10<P_{\mathrm{vd}}<30 \\ 0.6 & \text { if } P_{\mathrm{vd}}>30\end{cases}$

\section{Estimation of the probable vector density}

The number of female mosquitoes per person (MPP) has been used as vector density, and the reproduction number $R_{0}$ is directly proportional to the MPP under the assumption that the biting rate of the vector is steady in the following equation $[6,8]$.

$R_{0}=m a^{2} b c \exp \left(-\mu \tau_{\mathrm{e}}\right) / \mu \gamma$,

where $m$ is the MPP, $a$ is the daily biting rate of the mosquitoes, and $b$ and $c$ are the probabilities of viral transmission from an infected mosquito to a susceptible human, and from an infected human to mosquito, respectively [6]. Parameters $\mu, \gamma$, and $\tau_{e}$ are used as in Eq. (1). Estimated MPP is calculated from $R_{O i}$ using a Monte Carlo simulation function in Oracle Crystal Ball software (Oracle Corporation, Redwood Shores, CA, USA). Values of parameters used for calculating $R_{0 \mathrm{i}}$ and MPP are shown in Table 1. The goodness of fit of distributions was tested using Anderson-Darling statistics.

\section{Results}

The number of reported dengue cases and the date of the onset of symptoms (from August 12 to September 3, 2014) are shown in Fig. 1a. During this interval, 67 individuals with no history of overseas travel were reported in 12 prefectures. Among these 67, one case had not visited Yoyogi Park, and another lacked a date of onset; thus, 65 
Table 1 Parameters and parameter values

\begin{tabular}{llll}
\hline Parameter & Symbol & Value & Source references \\
\hline Host parameters & & & \\
Intrinsic incubation period & $\tau_{\mathrm{i}}$ & Gamma $(53.8,0.1)$ & {$[13]$} \\
$\quad$ Inverse of the duration of infectious period & $\gamma$ & $1 /$ Gamma $(25,0.2)$ & {$[13]$} \\
Vector parameters & & & \\
Extrinsic incubation period & $\tau_{\mathrm{e}}$ & Equation (2) & {$[1]$} \\
Daily mortality rate & $\mu$ & Equation $(3)$ & {$[7]$} \\
Daily biting rate of the mosquitoes & $a$ & 0.3 & {$[1]$} \\
Host and vector parameters & & & {$[1]$} \\
Transmission probability (vector to host) & $b$ & 0.46 & {$[1]$} \\
Transmission probability (host to vector) & $c$ & 0.83 & \\
\hline
\end{tabular}

Fig. 1 a Number of reported dengue cases on the day when onset of symptom was observed. b Daily number of new cases number of cases from August 12 to September 3, 2014. Dotted ellipse shows linear phase of initial epidemic plotted against the cumulative
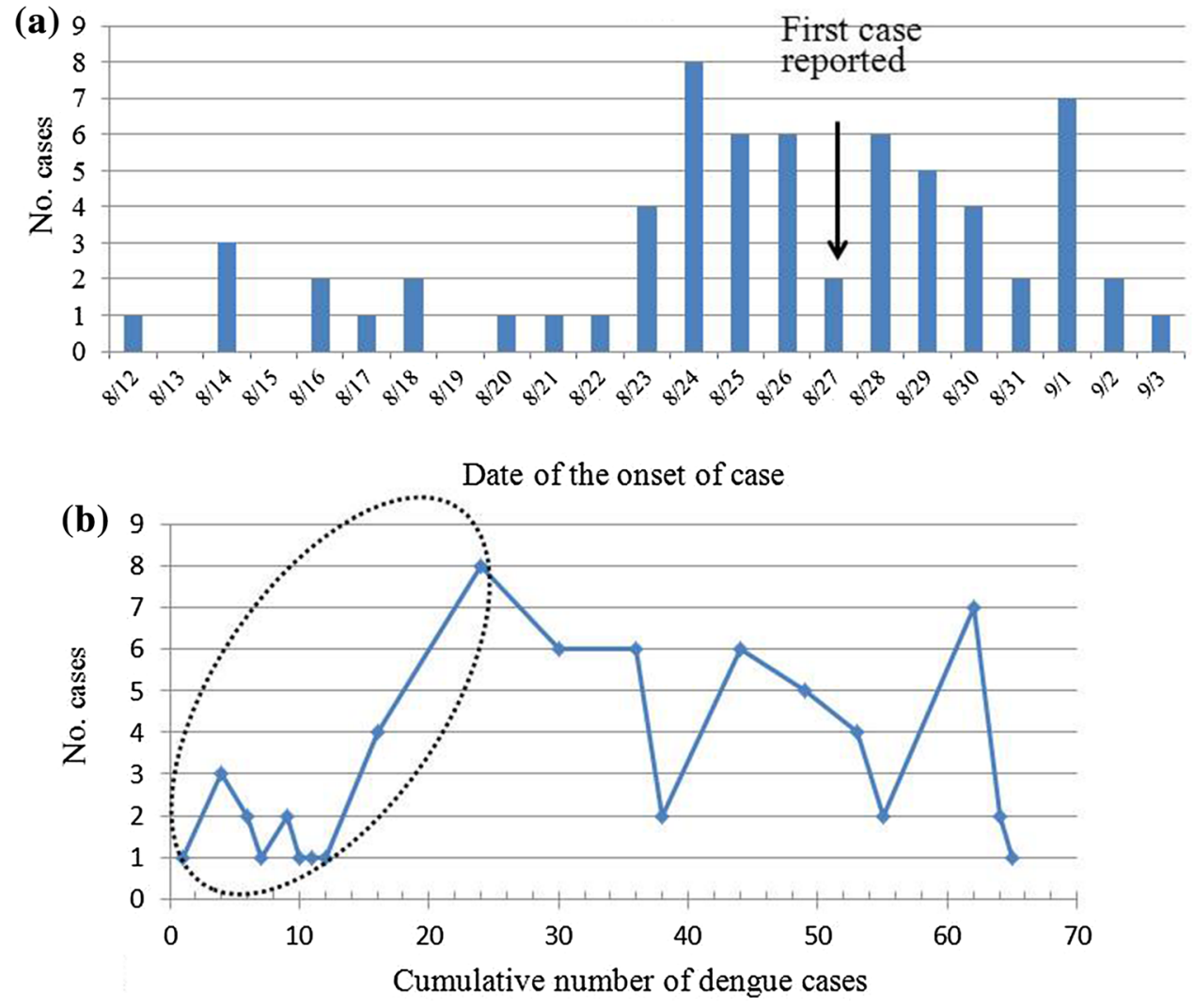

cases who visited Yoyogi Park were included in our analysis [4]. The resulting graph (plotting the daily number of new cases against the cumulative number of cases from August 12 to September 3, 2014) is shown in Fig. 1 b.

The cumulative number of cases on a logarithmic scale against the days from August 12 to September 3, 2014, turning point was observed on the 12th day. Thus, a linear increase up to the 12th day (accumulate case number is 24) was used for initial growth of the epidemic, and the parameter $\lambda$, obtained as the slope of linear phase of initial epidemic, was $0.261\left(R^{2}=0.581, p=0.01\right)$.
Daily mean and maximal temperatures, and daily mean relative humidity for each day from July 7 to August 16 (when the cases in the initial epidemic presumably were infected) are shown in Fig. 2 [9]. Mean of daily mean temperature and $\mathrm{RH}$ during this period were used to estimate vector parameters $\tau_{\mathrm{e}}$ and $\mu$. Estimated distribution $R_{0 \mathrm{i}}$ was fitted to a Gamma distribution using location parameter 4.25 , scale parameter 0.19 , and shape parameter 7.76 with median 7.78 and IQR (7.21-8.40). Estimated distribution of MPP was fitted to a normal distribution with mean 5.71 and standard deviation 0.53 . 
Fig. 2 Daily mean and maximal temperatures, and daily mean relative humidity for each day from July 7 to August 16,2014 , when cases in the initial epidemic are thought to have been infected. Mean of daily mean temperature and relative humidity during the period were used in the Eq. (2) and the Eq. (4)

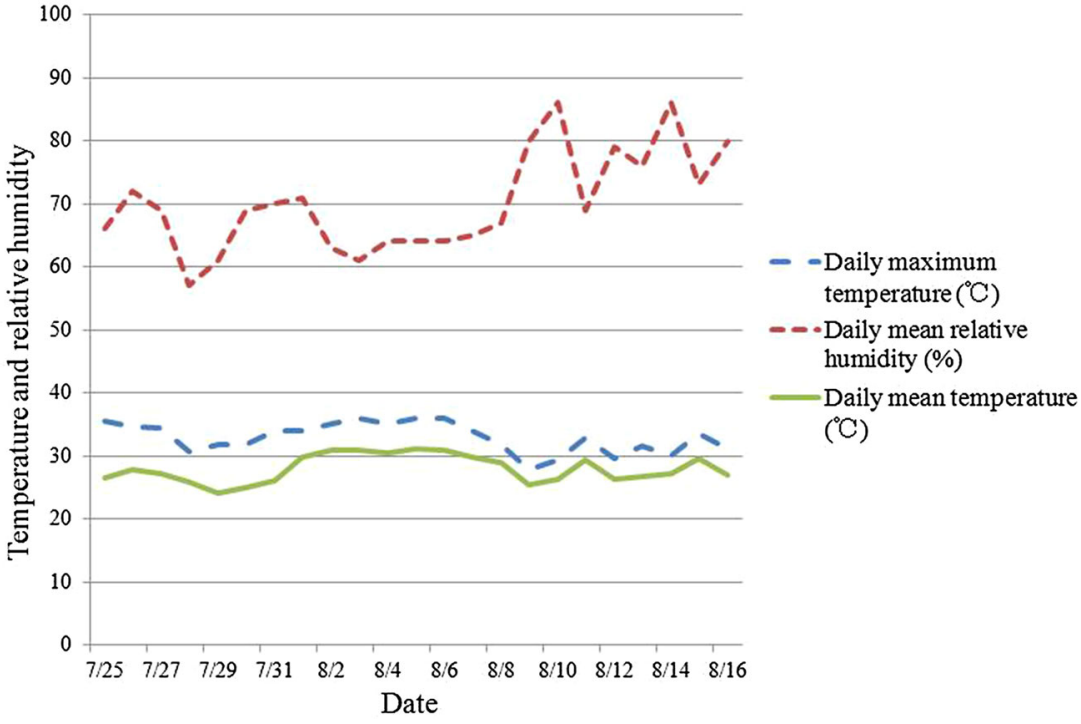

\section{Discussion}

From August 7, 2014, when the first illness onset was confirmed, through the end of October 2014, a total of 160 autochthonous dengue cases were reported. The majority of the cases [128/160 (80 \%)] were linked to Yoyogi Park, and the park was closed from September 4 to October 31 of that year. Arima et al. [3] summarized dengue cases from August to 17 September 2014. Kutsuna et al. [10] reported 19 cases of confirmed autochthonous dengue fever treated at the National Center for Global Health and Medicine during the interval from August 26 to September 22, 2014. Dengue virus serotype 1 (DENV 1) was confirmed in 14 of 19 cases, who were presumably exposed in Yoyogi Park. Sequencing of the strains from two of these 14 cases revealed that the dengue virus envelope protein encoded by each of these two strains was identical to that of a dengue virus strain isolated from the first patient in this outbreak in Japan. Their result suggested that a single strain may have been the source of the dengue outbreak in Yoyogi Park [10]. A male student in England traveled to Japan and stayed with a friend in Tokyo during the interval from July-September 2014; this individual developed dengue fever [11]. This individual stated that the house where he stayed in Japan is a 2-min walk from Yoyogi Park, and that he was bitten multiple times by mosquitoes there in late August, although he did not enter the park. This case showed the potential risk for travelers to become infected with dengue in a non-endemic area. The present work attempts to quantify the potential risk of dengue infection based on this spread of a local outbreak in a non-endemic country.

Though $50-80 \%$ of dengue virus infections can be asymptomatic [12]; Favier et al. [7] and Chowell et al. [13] reported that the estimation method used in this study had robust to the type of underreporting that is generated by asymptomatic or mild cases of dengue as long as the reporting rate is "constant" during the initial epidemic growth.

Based on an $R_{0}$ cut-off of $\geq 1$ (in non-endemic conditions), Oki and Yamamoto [8] estimated threshold MPPs of 0.36 and 4.47 for the most favorable and least favorable conditions, respectively, for disease spread in the 2000s. Given that the MPP value 5.71 in this study was nearer to the value under the least favorable conditions for disease spread, the epidemic considered here may have included higher blood feeding frequency and/or longer vector survival.

Tsuda et al. [14] collected A. albopictus mosquitoes from Yoyogi Park on 4 and 5 September, 2014, and estimated that the overall mean density of biting mosquitoes was $7.13 /$ person/8 min. A previous investigation of the arrival patterns of A. albopictus on a person during 30-min collection intervals showed that 56 and $68 \%$ of the total female mosquitoes were collected during the first 5 and $10 \mathrm{~min}$ of the mosquito collection, respectively [15]. Thus, those authors considered that an 8-min mosquito collection period (rather than the standard 30-min interval) should be sufficient to obtain a reliable estimate of biting density. They also found that the frequency of infected mosquitos was higher in areas in which the mosquito density was higher than the overall mean mosquito density [14].

$R_{O}$ has been estimated as 2.0-10.3 in Brazil [7], 1.89-2.23 in Singapore [16], and 3.93-4.67 in Taiwan [17] in previous reports of dengue outbreaks in areas where dengue is vectored by $A$. aegypti. As sample size in this study is smaller than those for other studies, it has difficulty in comparing the result with other results, directly. Though 
the available data in this study are small and uncertainties are large, estimated MPP is nearer to the overall mean density of biting mosquitoes from the mosquito collection. Thus, the author considers that the estimated reproduction number $R_{0 \mathrm{i}}$ is not strongly biased.

The larger value of $R_{0 \mathrm{i}}$ with median 7.78 in the present study may reflect the fact that 38 of our cases were in their teens or $20 \mathrm{~s}$, and stayed in the park for many hours. Additionally, higher daily mean temperature and $\mathrm{RH}$ were observed in the beginning and middle of August, respectively; the elevated temperature and humidity may have increased the growth and biting activity of the mosquitoes.

This epidemic was followed by an August park event that was attended by people from across the nation (http:// www.yoyogipark.info/ad2014/). Cases from 18 separate prefectures proved to be attendees of this event, suggesting that such assemblies may have spread dengue nationwide.

The present study has the limitation that asymptomatic cases were not considered. Dengue virus infection includes the asymptomatic cases as well as cases that are characterized by undifferentiated fever. A review by Chastel [18] examined the role of asymptomatic cases of dengue in the introduction and spread of dengue viruses in non-endemic regions. Asymptomatic cases are much more frequent than the symptomatic ones, but the ratio of asymptomatic/ symptomatic varied from 1.1 to 13.0. Viremia is considered to be lower and shorter in duration in asymptomatic persons than in symptomatic ones, and asymptomatic persons have less infectivity to competent mosquitoes. Nonetheless, asymptomatic dengue virus infections are considered to serve as potential sources of epidemics. However, this speculation has not been proven; active surveillance will be needed to assess the frequency and role of asymptomatic dengue cases.

Daily number of infected cases had been potentially underreported in this study; the estimated reproduction number and probable vector density may lead to underestimation of true infection risk. As the date of adult vector surveillance occurred later than the presumed date of infection, speculation regarding the potential relationships between the density and geographical spread of infected vectors and dengue incidence was necessarily tentative.

Both estimated reproduction number and vector density per person at the onset of the epidemic were higher than previously reported values. These results indicate the potential for dengue outbreaks in places with elevated vector density per person, even in dengue non-endemic countries. Several people also became infected with dengue in other parks located 2-8 km away from Yoyogi Park in Tokyo. Although data on the flight range of Aedes albopictus in the vicinity of park is limited, there remains a possibility that human movement was involved in this epidemic. To investigate the cause of this outbreak, further studies will be needed, including assessments of social, behavioral, and environmental factors that may have contributed to this epidemic by altering host and vector conditions in the park.

Acknowledgments The author thanks the organizer and participants of 2014 Infectious Disease Modeling Course for discussions regarding future dengue epidemics in Japan. This work was partially supported by JSPS KAKENHI Grant Number 25460631.

\section{Compliance with ethical standards}

Conflict of interest There are no other conflicts of interest to declare.

\section{References}

1. Oki M, Yamamoto T. Simulation of the probable vector density that caused the Nagasaki dengue outbreak vectored by Aedes albopictus in 1942. Epidemiol Infect. 2013;141:2612-22.

2. Schmidt-Chanasit J, Emmerich P, Tappe D, Gunther S, Schmidt $\mathrm{S}$, Wolff D et al. Autochthonous dengue virus infection in Japan imported into Germany, September 2013. Euro Surveill. 2014; 19(3).

3. Arima $\mathrm{Y}$, Matsui $\mathrm{T}$, Shimada $\mathrm{T}$, Ishikane $\mathrm{M}$, Kawabata $\mathrm{K}$, Sunagawa $\mathrm{T}$, et al. Ongoing local transmission of dengue in Japan, August to September. West Pac Surveill Respon J. 2014; doi:10.5365/wpsar.2014.5.3.007.

4. Reports about dengue fever from Ministry of Health and Welfare, Japan (in Japanese). http://www.mhlw.go.jp/bunya/kenkou/kek kaku-kansenshou19/dengue_fever.html. Accessed 20 Nov 2014.

5. Massad E, Coutinho FA, Burattini MN, Lopez LF. The risk of yellow fever in a dengue-infested area. Trans R Soc Trop Med Hyg. 2001;95:370-4.

6. Degallier N, Favier C, Boulanger JP, Menkes C. Imported and autochthonous cases in the dynamics of dengue epidemics in Brazil. Rev Saude Publica. 2009;43(1):1-7.

7. Favier C, Degallier N, Rosa-Freitas MG, Boulanger JP, Costa Lima JR, Luitgards-Moura JF, et al. Early determination of the reproductive number for vector-borne diseases: the case of dengue in Brazil. Trop Med Int Health. 2006;11:332-40.

8. Oki M, Yamamoto T. Climate change, population immunity, and hyperendemicity in the transmission threshold of dengue. PLoS One. 2012;7(10):e48258. doi:10.1371/journal.pone.0048258.

9. Japan Meteorological Agency. http://www.data.jma.go.jp/obd/ stats/data/mdrr/index.html Accessed 20 Nov 2014.

10. Kutsuna S, Kato Y, Moi ML, Kotaki A, Ota M, Shinohara K, et al. Autochthonous dengue Fever, Tokyo, Japan, 2014. Emerg Infect Dis. 2015;21(3):517-20. doi:10.3201/eid2103/141662.

11. Kojima G. Autochthonous Dengue Fever Imported to England from Japan, 2014. Emerg Infect Dis. 2015;21(1):182-4. doi:10. 3201/eid2101.141581.

12. Burke DS, Nisalak A, Johnson DE, Scott RM. A prospective study of dengue infections in Bangkok. Am J Trop Med Hyg. 1988;38(1):172-80.

13. Chowell G, Diaz-Dueñas P, Miller JC, Alcazar-Velazco A, Hyman JM, Fenimore PW, et al. Estimation of the reproduction number of dengue fever from spatial epidemic data. Math Biosci. 2007;208:571-89.

14. Tsuda Y, Maekawa Y, Ogawa K, Itokawa K, Komagata O, Sasaki T, Isawa H, Tomita T, Sawabe K. Biting density and distribution of Aedes albopictus during the September 2014 outbreak of dengue fever in Yoyogi Park and the vicinity in Tokyo 
Metropolis, Japan. Jpn J Infect Dis. 2015. doi:10.7883/yoken. JJID.2014.576.

15. Tsuda Y, Kim KS. Ecology of mosquitoes inhabiting a park in urban Tokyo, Japan: density of biting Aedes albopictus and laboratory estimations of the residual longevity. Med Entomol Zool. 2012;63:223-30.

16. Hsieh $\mathrm{YH}$, Ma S. Intervention measures, turning point, and reproduction number for dengue, Singapore, 2005. Am J Trop Med Hyg. 2009;80:66-71.
17. Hsieh YH, Chen CW. Turning points, reproduction number, and impact of climatological events for multi-wave dengue outbreaks. Trop Med Int Health. 2009;14:628-38.

18. Chastel C. Eventual role of asymptomatic cases of dengue for the introduction and spread of dengue viruses in non-endemic regions. Front Physiol. 2012;3:70. doi:10.3389/fphys.2012. 00070. 